EPJ Web of Conferences 21, 10001 (2012)

DOI: 10.1051/epjconf/20122110001

(C) Owned by the authors, published by EDP Sciences, 2012

\title{
Effects of fissioning nuclei distributions on fragment mass distributions for high energy fission
}

\author{
E Andrade-II ${ }^{1}$, J C M Menezes ${ }^{2}$, S B Duarte ${ }^{3}$, F Garcia $^{2}$, P C R Rossi ${ }^{4}$, O A P Tavares ${ }^{3}$, and A \\ Deppman $^{1, \text { a }}$ \\ 1 Instituto de Fisica, Universidade de Sao Paulo - IFUSP, Rua do Matao, Travessa R 187, 05508-900 \\ Sao Paulo-SP, Brazil \\ 2 Departamento de Ciencias Exatas e Tecnologicas, Universidade Estadual de Santa Cruz-UESC, \\ Rodovia Ilheus-Itabuna km16, 45650-000 Ilheus-Ba, Brazil \\ 3 Centro Brasileiro de Pesquisas Fisicas-CBPF/MCT, Rua Dr. Xavier Sigaud, 150, 22290-180, Rio de \\ Janeiro-RJ, Brazil \\ 4 Divisao de Reatores, Instituto de Pesquisas Energeticas e Nucleares-IPEN/CNEN-SP, Rua do Matao, \\ Travessa R 400, 05508-900 São Paulo-SP, Brazil
}

\begin{abstract}
We study the effects of fissioning nuclei mass- and energy-distributions on the formation of fragments for fission induced by high energy probes. A Monte Carlo code called CRISP was used for obtaining mass distributions and spectra of the fissioning nuclei for reactions induced by $660 \mathrm{MeV}$ protons on ${ }^{241} \mathrm{Am}$ and on ${ }^{239} \mathrm{~Np}$, by $500 \mathrm{MeV}$ protons on ${ }^{208} \mathrm{~Pb}$, and by Bremsstrahlung photons with end-point energies at $50 \mathrm{MeV}$ and $3500 \mathrm{MeV}$ on ${ }^{238} \mathrm{U}$. The results show that even at high excitation energies, asymmetric fission may still contribute significantly to the fission cross section of actinide nuclei, while it is the dominante mode in the case of lead. However, more precise data for high energy fission on actinide are necessary in order to allow definite conclusions.
\end{abstract}

\section{Introduction}

The well-known multimode approach to calculate fission fragments masses is an interesting way to study fission dynamics. According to this model, fragment mass distributions are given by a sum of gaussian distributuions corresponding to the different modes through which fission may take place, and we can write the mass distribution as [1]

$$
\begin{array}{r}
\sigma(A, Z)=\left\{\sum_{i}\left[\frac{K_{i}^{\mathrm{L}}}{\sqrt{2 \pi} \Gamma_{i}^{\mathrm{L}}} \exp \left(-\frac{\left(A-A_{i}^{\mathrm{L}}\right)^{2}}{2\left(\Gamma_{i}^{\mathrm{L}}\right)^{2}}\right)+\frac{K_{i}^{\mathrm{H}}}{\sqrt{2 \pi} \Gamma_{i}^{\mathrm{H}}} \exp \left(-\frac{\left(A-A_{i}^{\mathrm{H}}\right)^{2}}{2\left(\Gamma_{i}^{\mathrm{H}}\right)^{2}}\right)\right]+\right. \\
\left.\frac{K_{\mathrm{S}}}{\sqrt{2 \pi} \Gamma_{\mathrm{S}}} \exp \left(-\frac{\left(A-A_{\mathrm{S}}\right)^{2}}{2\left(\Gamma_{\mathrm{S}}\right)^{2}}\right)\right\} \frac{1}{\sqrt{2 \pi} \Gamma_{\mathrm{Z}}} \exp \left(-\frac{\left(Z-\overline{Z_{0}}\right)^{2}}{2 \Gamma_{\mathrm{Z}}^{2}}\right)
\end{array}
$$

where the summation runs over the asymmetric modes. The parameters for the symmetric mode are $K_{\mathrm{S}}, A_{\mathrm{S}}$ and $\Gamma_{\mathrm{S}}$, while $K_{i}^{\mathrm{H}(\mathrm{L})}, A_{i}^{\mathrm{H}(\mathrm{L})}$ and $\Gamma_{i}^{\mathrm{H}(\mathrm{L})}$ are the parameters for the heavy (light) fragment produced in the asymmetric mode $i$. For the atomic number distribution the parametrization used is [2]

$$
\overline{Z_{0}}=\mu_{1}+\mu_{2} A
$$

for the most probable atomic number of the fragment, and

\footnotetext{
a e-mail: deppman@if.usp.br
}

This is an Open Access article distributed under the terms of the Creative Commons Attribution-Noncommercial License 3.0, which permits unrestricted use, distribution, and reproduction in any noncommercial medium, provided the original work is properly cited. 


$$
\Gamma_{\mathrm{Z}}=v_{1}+v_{2} A
$$

for the width of the atomic number distribution. $\mu_{1}, \mu_{2}, v_{1}$ and $v_{2}$ are fitting parameters. In the discussion below the dependence of distributions on the atomic number $\mathrm{Z}$ will not be relevant.

Yield, position and width parameters for each mode in equation (1) and $\mu_{1}, \mu_{2}, v_{1}$ and $v_{2}$ in (2) and (3) are usually considered free parameters for fitting procedure. This method has been used for describing spontaneous fission [3], low-energy induced fission [4-6], fission induced by thermal-neutrons [7-9] and $12 \mathrm{MeV}$ protons [10], and even for fission induced by intermediate energy probes such as $190 \mathrm{MeV}$ protons [11], neutrons at energies up to $200 \mathrm{MeV}$ [12], and also by heavy-ions [13,14]. A systematic study of the values obtained for the parameters in formula (1) by fitting to experimental data for spontaneous or low energy fission of several nuclei was performed by Böckstiegel et al.[6], showing that those parameters can be described by smooth functions of the fissioning nucleus mass number. More recently, equation 1 has been applied for describing ${ }^{238} \mathrm{U}$ fission induced by photons from Bremsstrahlung [15] with end-point energies of $50 \mathrm{MeV}$ and $3500 \mathrm{MeV}$, and fission induced by $660 \mathrm{MeV}$ protons on ${ }^{241} \mathrm{Am}$ and ${ }^{237} \mathrm{~Np}[16]$.

In recent papers $[17,18]$ we discussed about the effects of the mass- and energy-distributions on the final fragment mass distributions. We have shown that in fission induced by high energy probes, as protons and photons, the fissioning nuclei are quite different from the target nucleus due to the emission of particles during intranuclear cascade and during the evaporation steps before fission occurs. In fact, we show the mass distributions of the fissioning nuclei for fission induced by $660 \mathrm{MeV}$ on ${ }^{241} \mathrm{Am}$ and on ${ }^{239} \mathrm{~Np}$ and by Bremmstrahlung photons with endpoint energies of $50 \mathrm{MeV}$ and $3500 \mathrm{MeV}$ on ${ }^{238} \mathrm{U}$ in Figure 1, and for fission induced by $500 \mathrm{MeV}$ protons on ${ }^{208} \mathrm{~Pb}$ in Figure 1

It is easy to observe the large mass distribution for the fissioning nuclei, with masses that can be rather smaller than the target nuclei one. Also, we notice that these mass distribution depends on the probe used. In fact the distributions for fission induced by protons on Am and Np are larger than that for the case of photons on U. These differents can be attributed to the dominance of low energy photons in the Bremmstrahlung spectrum, but also to the different reaction mechanisms for protons and photons. Since photon-nucleus interaction is weak as compared to proton-nucleus interaction, the former interacts in the whole nuclear volume, while the later interacts on the nuclear surface.

Comparing Figures 1 and 2 we can conclude that there is also a dependence of the mass distributions on the target. Even for similar energies (500 MeV and $600 \mathrm{MeV})$, the distribution obtained in the case of $\mathrm{Pb}$ is narrower than those for $\mathrm{Am}$ and $\mathrm{Np}$. We can understand these results if we recall that fission probabilities are much higher for nuclei with masses above $A \approx 230$, and decreases very fast as $A$ decreases below 230(see, for instance, Ref. [19]). Therefore, in the reactions on Np, Am or $\mathrm{U}$ there is a considerable probability for fission even after the emission of several nucleons during the intranuclear and evaporation steps, while in the reactions on $\mathrm{Pb}$ the fission probabilities decreases very fast as nucleons are emitted. Thus, the mass distribution of the fissioning nuclei in the case of $\mathrm{Pb}$ is dominated by fission probability, while in the case of actinide nuclei it is dominated by the nucleon emission.

Therefore, at intermediate and high energies, the fragment mass distributions are folded into the fissioning-system mass distributions. A more realistic calculation of fission fragment mass distribution must take into account the broad mass distributions of the fissioning nuclei. As it will be shown in the following, these distributions not only affect the calculated fragment mass distributions, but also are dependent on the probe used and on its incident energy.

Even after the emission of several particles during the intranuclear cascade and evaporation processes, the fissioning nucleus is higly excited. In Figures 3 and 4 we observe that for reactions induced by $660 \mathrm{MeV}$ or $500 \mathrm{MeV}$ protons the fission occurs at excitation energies around $150 \mathrm{MeV}$. For fission induced by Bremmstrahlung the excitantion energies are lower due to the dominance of low energy photons in the Bremmstrahlung spectrum, but in the case of end-point energy at $3500 \mathrm{MeV}$, it is still possible to find fissioning nuclei at excitantion energies above $200 \mathrm{MeV}$.

In $[17,18]$ we have shown how to unfold the contributions due to fission modes and to fissioningnuclei distribution in the fragment-mass distribution by using Monte Carlo method. CRISP is a Monte Carlo code for simulating nuclear reactions [20] that uses a two step process. First, an intranuclear 


\section{CNR*11}

cascade is simulated following a time-ordered sequence of collisions in a many-body system [21,22], and when the intranuclear cascade is finished the evaporation of nucleons and alpha-particles starts in competition with fission [23].

In the simulation, reactions can be initiated by intermediate and high energy protons [22] or photons [24-26]. It has been extended to energies up to $3.5 \mathrm{GeV}$ [27], and it was shown that the CRISP code can give good results for total photonuclear absorption cross sections from approximately 50 $\mathrm{MeV}$, where the quasi-deuteron absorption mechanism is dominant, up to $3.5 \mathrm{GeV}$, where the socalled photon-hadronization mechanism is dominant, leading to a shadowing effect in the cross section [27]. One important feature in the simulation of the intranuclear cascade is the Pauli blocking mechanism, which avoids violation of the Pauli principle. In CRISP a strict verification of this principle is performed at each step of the cascade, resulting in a more realistic simulation of the process. The advantages of such an approach have been discussed elsewhere (see [20] and references therein).

In the evaporation/fission competition that follows intranuclear cascade, Weisskopf's model is adopted for calculating the branching ratios of the evaporating channels, which includes evaporation of neutrons, protons and alpha-particles $[23,25,26]$ and Bohr-Wheeler-model is adopted for fission. The code has provided photofission cross sections in good agreement with experimental data [20]. The CRISP code has already been used for evaluating mass distributions of fragments for fission induced by photons at intermediate energies [28], and to calculate spallation yields and neutron multiplicities for reactions induced by high energy protons [29], giving results in good agreement with experimental data. Also, the code has already been used in studies of the ADS (Accelerator Driving System) nuclear-reactors [29-32]. Recently the CRISP code has been used to study the effects of final state interaction in the decay of hypernuclei $[33,34]$.

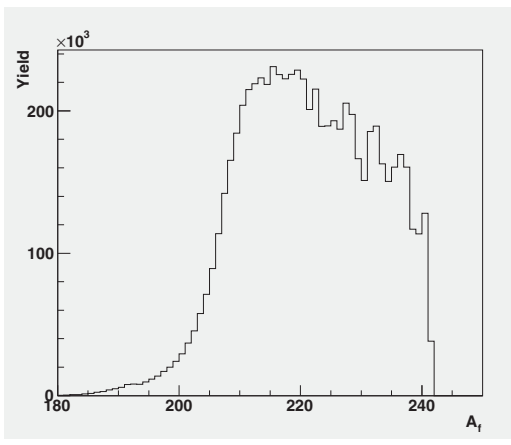

(a)

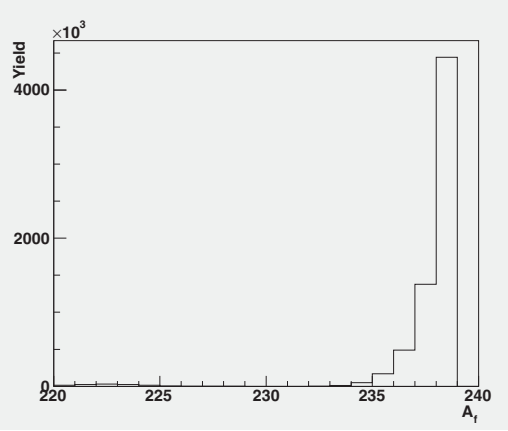

(c)

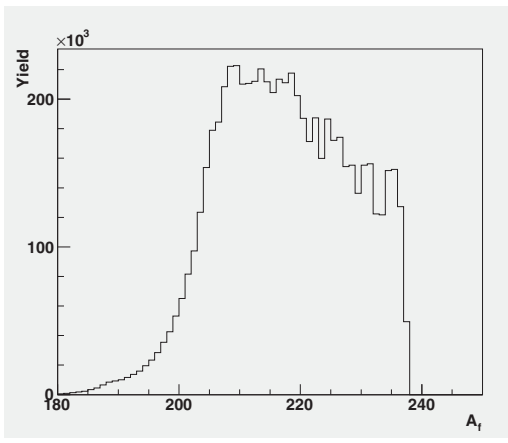

(b)

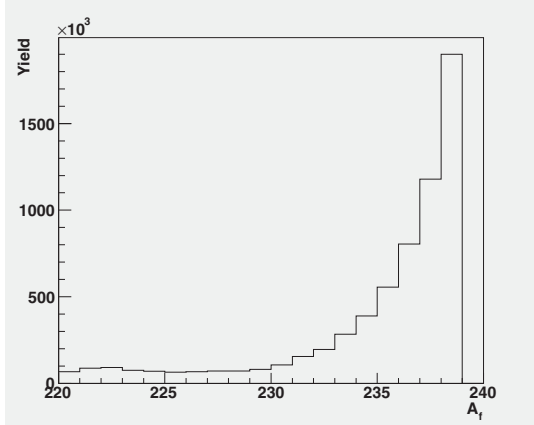

(d)

Fig. 1. Mass distribution of the fissioning nucleus in reactions induced by $660 \mathrm{MeV}$ protons on ${ }^{241} \mathrm{Am}$ (a) and ${ }^{237} \mathrm{~Np}$ (b) target nuclei and in fission of ${ }^{238} \mathrm{U}$ induced by Bremsstrahlung with end-point energies of $50 \mathrm{MeV}$ (c) and $3500 \mathrm{MeV}$ (d) as obtained by the CRISP code. 


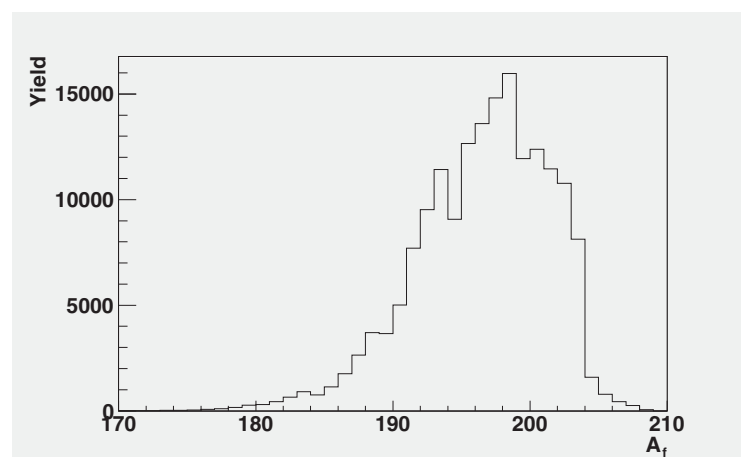

Fig. 2. Mass distribution of the fissioning nucleus in reactions induced by $500 \mathrm{MeV}$ protons on ${ }^{208} \mathrm{~Pb}$ target nucleus as obtained by the CRISP code.

Table 1. Values for some of the relevant parameters in the multimode formula for the fission-fragment mass distributions. The low-energy parameters are those obtained from the systematic study for spontaneous and lowenergy fission[6] (here the errors are estimated range); those for ${ }^{241} \mathrm{Am}$ and ${ }^{237} \mathrm{~Np}$ are results of fittings for 660 $\mathrm{MeV}$ protons[16], and the parameters for uranium are those obtained from fittings to data of fission induced by Bremsstrahlung photons with end-point energies of $50 \mathrm{MeV}$ and $3500 \mathrm{MeV}$ [15].

\begin{tabular}{llllll}
\hline parameter & low-energy & ${ }^{241} \mathrm{Am}$ & ${ }^{237} \mathrm{~Np}$ & ${ }^{238} \mathrm{U}(50 \mathrm{MeV})$ & ${ }^{238} \mathrm{U}(3500 \mathrm{MeV})$ \\
\hline$\Gamma_{\mathrm{S}}$ & $10.0 \pm 2$ & $15.0 \pm 0.9$ & $13.7 \pm 1.0$ & $12.0 \pm 0.48$ & $12.0 \pm 0.48$ \\
$A_{1}^{\mathrm{H}}$ & $135.0 \pm 1$ & $133.5 \pm 0.72$ & $133.0 \pm 0.98$ & $133.32 \pm 1.03$ & $133.32 \pm 1.03$ \\
$\Gamma_{1}^{\mathrm{H}}$ & $3.75 \pm 2$ & $4.2 \pm 0.5$ & $4.5 \pm 0.4$ & $3.54 \pm 0.4$ & $3.54 \pm 0.4$ \\
$A_{2}^{\mathrm{H}}$ & $141.0 \pm 2$ & $139.0 \pm 1.17$ & $138.0 \pm 1.03$ & $137.5 \pm 1.41$ & $137.5 \pm 1.41$ \\
$\Gamma_{2}^{\mathrm{H}}$ & $5.0 \pm 1$ & $7.0 \pm 0.5$ & $6.5 \pm 0.6$ & $6.0 \pm 0.21$ & $6.0 \pm 0.21$ \\
\hline \multicolumn{5}{c}{ Type 2} & calculation \\
\hline$K_{\mathrm{S}}(m b)$ & - & $2970.0 \pm 20.5$ & $2590.0 \pm 23.3$ & $23.75 \pm 0.7$ & $23.75 \pm 0.7$ \\
$K_{1}(m b)$ & - & $45.8 \pm 0.2$ & $49.0 \pm 0.3$ & $7.5 \pm 0.04$ & $7.5 \pm 0.04$ \\
$K_{2}(m b)$ & - & $220.5 \pm 1.5$ & $252.0 \pm 1.3$ & $140.0 \pm 7.2$ & $140.0 \pm 7.2$ \\
\hline
\end{tabular}

In the systematic study carried out by Böckstiegel et al.[6] for spontaneous fission and low-energy reactions, the fissioning nuclei can be considered identical to the initial (or target) nuclei, and thus the problem due to fissioning nuclei properties does not arise. On the other hand, fittings to fission data obtained for high energies lead to values of the parameters that deviate from the systematic at lowenergy fission. Based on this systematics for low-energy or spontaneous fission, one gets the estimated values for the relevant parameters in (4), which are shown in Table 1 (upper part), and compare them to the corresponding values obtained by fittings of high-energy fission data. One can see that for high energy reactions the width obtained for the symmetric channel is systematically higher than those obtained with low energy or spontaneous fission data, and the position for the peak in the asymmetric fission masses are shifted down with respect to the systematic values. This is an evidence of the effect of fissioning system distributions on the values obtained in the multimode analysis.

Using the CRISP code it is possible to separate the effects of the fission-channel width and those of the mass-distribution of the fissioning nucleus because the fission process is considered for each individual fissioning nucleus. To this end we rewrite Equation 1 in the form

$$
p(A, Z)=\left\{\sum_{i} \frac{p_{i}}{\sqrt{2 \pi} \Gamma_{i}} \exp \left[-\frac{\left(A-A_{i}\right)^{2}}{2 \Gamma_{i}^{2}}\right]\right\} \frac{1}{\sqrt{2 \pi} \Gamma_{\mathrm{Z}}} \exp \left[-\frac{\left(Z-\overline{Z_{0}}\right)^{2}}{2 \Gamma_{\mathrm{Z}}^{2}}\right]
$$




\section{CNR*11}

where $p_{i}$ is the probability that fission occurs through the $i t h$ mode which is related to the intensities $K_{i}$ by the relation

$$
p_{i}=\frac{K_{i}}{\sum_{i} K_{i}}
$$

the index $i=S, 1,2$ corresponding to the modes $S L, S 1$ and $S 2$, respectively. The light fragments are obtained according to $A^{\mathrm{L}}=A_{\mathrm{f}}-A^{\mathrm{H}}$ and $Z^{\mathrm{L}}=\overline{Z_{0}}-Z^{\mathrm{H}}$. The $Z$ dependence of the probability is explicitly shown in (4) for the sake of completeness only, since it will not be relevant in the results presented in this work. Notice that in the expression (4) the symmetric fragment mass, $A_{\mathrm{S}}$, is no more a free parameter, but it is completely determined by simulations with the CRISP code till the fission point.
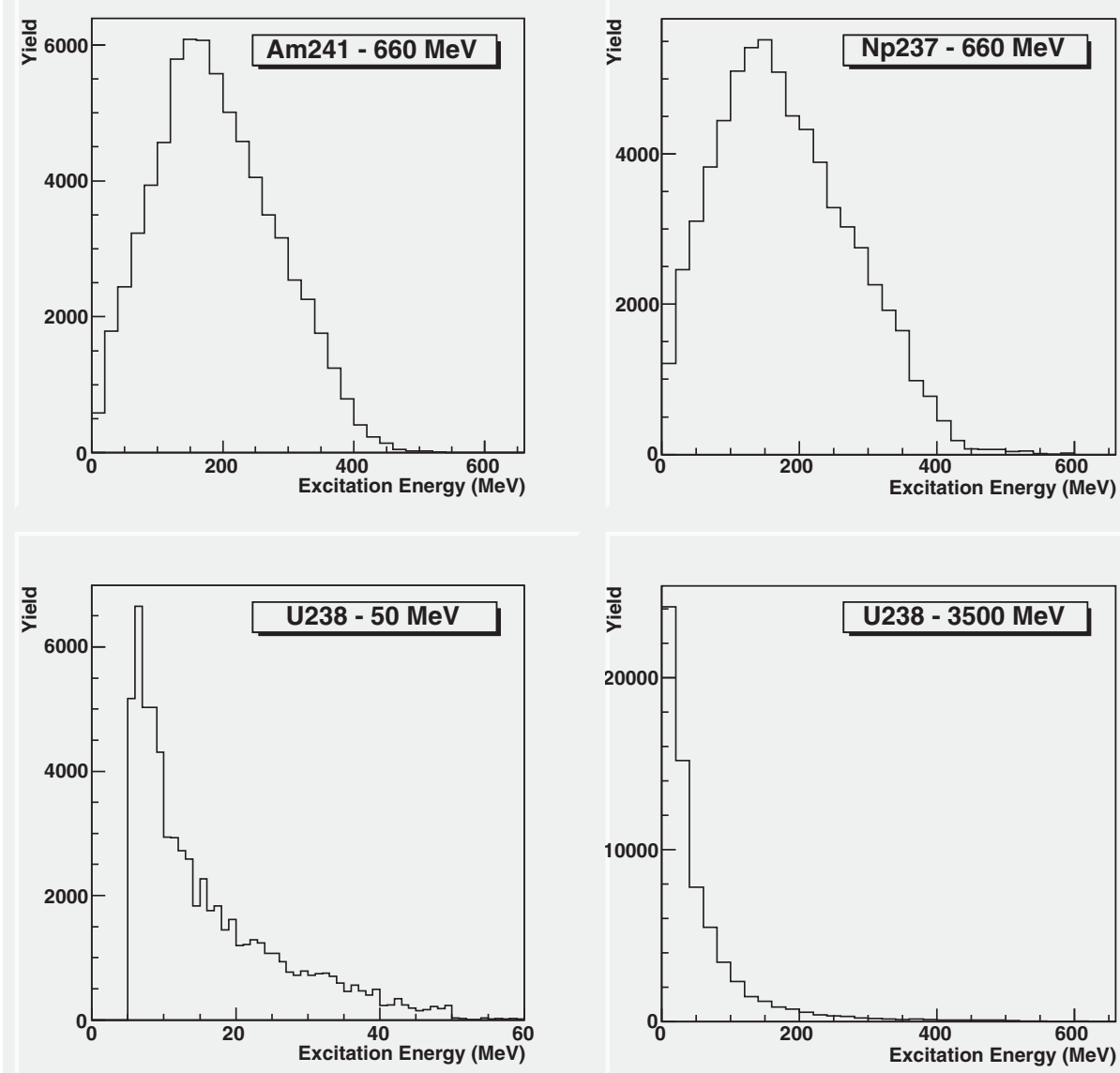

Fig. 3. Excitation energy distributions of the fissioning nuclei obtained with the CRISP code for different fission reactions of heavy nuclei as indicated.

For the determination of the fission fragments masses it is necessary to attribute values for the parameters used in the multimode approach, which is not a trivial problem for high energy induced fission. As observed in Figure 1, the mass number of fissioning nuclei varies over a broad range, and the multimode parameters that appear in equation (4) are not determined for all of them. Also, as shown in Figure 3, the excitation energy of the fissioning nuclei are in many cases around hundreds of $\mathrm{MeV}$, and the parameters are not determined at these energies. 


\section{EPJ Web of Conferences}

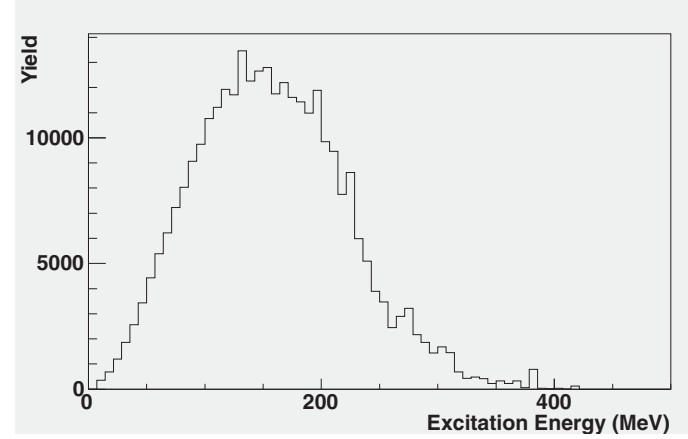

Fig. 4. Excitation energy of fissioning nuclei for $500 \mathrm{MeV}$ on ${ }^{208} \mathrm{~Pb}$

In the present work we extend the analysis performed in Ref [17] and discuss also the possibility of symmetric fission dominance at high energies. It was believed that at low energies fission should be predominantly asymmetric, while at high energies the symmetric mode should be dominant because at these energies the effects due to nuclear structure would be washed out. However, Siegler et al. [35] showed that there is an important contribution of the symmetric mode even at energies around $5 \mathrm{MeV}$. But it was still argued that at energies above $\sim 50 \mathrm{MeV}$ only the symmetric mode should be relevant. Therefore the first type of calculations performed here (Type 1) assumes that $p_{\mathrm{S}}=1, p_{1}=p_{2}=0$. For the other parameters, the values from the systematics [6] are used. Following [17], the second calculation (Type 2) is performed by using for $K_{\mathrm{S}}, K_{1}$ and $K_{2}$ the values found by Demekhina et al. and Karapetyan et al. $[15,16]$, presented in Table 1 (lower part), to calculate the corresponding probabilities through equation (5), while keeping all other parameters unchanged. Finally, a third calculation (Type $3)$ is performed considering the probabilities $p_{(\mathrm{S}, 1,2)}$ as gaussian functions of the fissioning nucleus mass number, which are obtained by fitting the systematic values from Böckstiegel et al. [6] and using linear extrapolation to lower masses $(A<220)$. Again all other parameters are kept unchanged as in the other calculations.

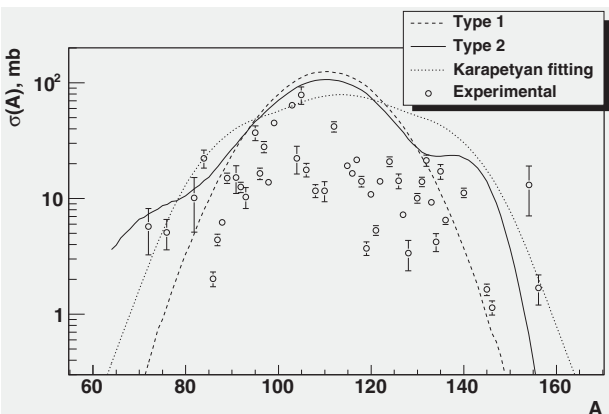

(a)

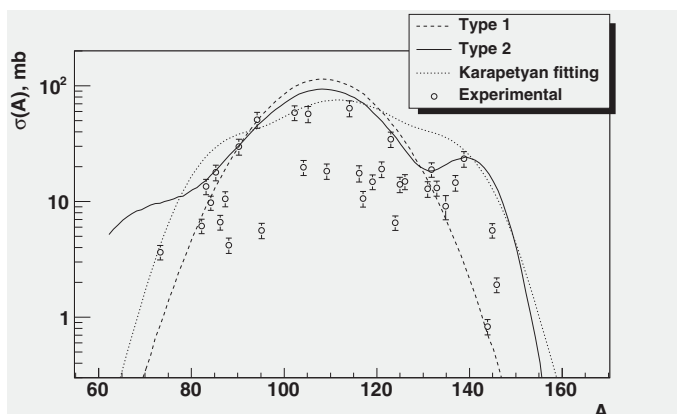

(b)

Fig. 5. Fragment-mass distribution for fission induced by $660 \mathrm{MeV}$ protons on ${ }^{241} \mathrm{Am}$ (a) and ${ }^{237} \mathrm{~Np}$ (b) targets considering calculation Type 1 (--) with values from the systematics [6] for the symmetric mode only and Type 2 (一) using the values for $K_{\mathrm{S}}, K_{1}$ and $K_{2}$ presented in Table 1 (lower part). Best fit found in [16] are represented by the dotted line (...). All results are compared with experimental data (o) [16].

In Figures 5 and 6 the results obtained are shown and are compared to experimental data and to the fitted distributions from $[15,16]$. It is possible to observe that for all reactions studied here the simple assumption that the symmetric mode is dominant above $\sim 50 \mathrm{MeV}$ (Type 1) results in distributions that are in disagreement with experiments. Type 2 and Type 3 calculations are quite different from the 


\section{CNR*11}

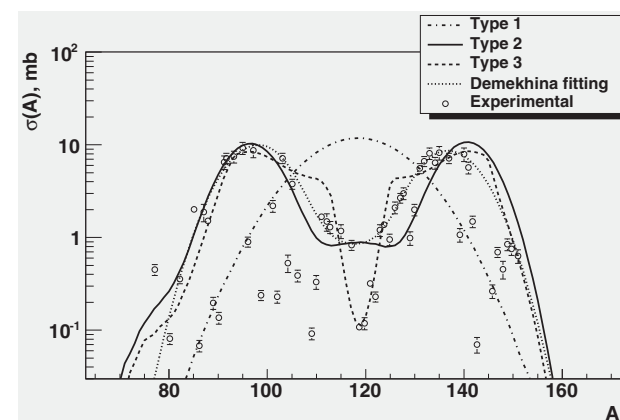

(a)

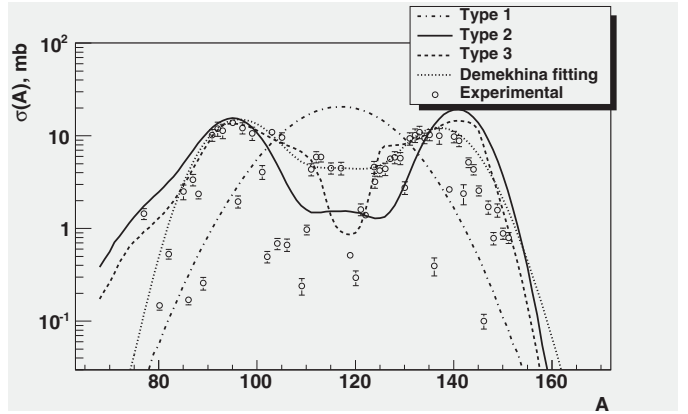

(b)

Fig. 6. Fragment-mass distribution for fission of ${ }^{238} \mathrm{U}$ induced by bremmstrahlung of $50 \mathrm{MeV}$ (a) and $3500 \mathrm{MeV}$ (b) end-point energies considering calculation Type 1 (.-.. .) with values from the systematics [6] for the symmetric mode only, Type $2(-)$ using the values for $K_{S}, K_{1}$ and $K_{2}$ presented in Table 1 (lower part) and Type 3 (--) performed considering the probabilities $p_{(\mathrm{S}, 1,2)}$ as gaussian functions of the fissioning nucleus mass number. The dotted line (...) represents the best fit of the formula (1) as done by Demekhina et al. [15] and open circules (o) are their experimental results.

calculations according to references $[15,16]$. This result shows that the effects of the fissioning nucleus mass distributions are significant, and that it must be included in a realistic calculation.

The comparison with the experimental data shows that the calculations describe correctly the position and width of the fragment mass distributions. More precise data would be useful for extracting physical information about the fission process.

Precise measurements of fragment mass distributions were obtained for fission induced by high energy protons at GSI [36]. Now we apply the same method to describe the experimental results obtained for the reaction $\mathrm{p}(500 \mathrm{MeV})+{ }^{208} \mathrm{~Pb}$. Unfortunately, there is not any systematic study of the multimodal parameters in the mass region of interest in this case, therefore we suppose that the symmetric fission mode is dominant.

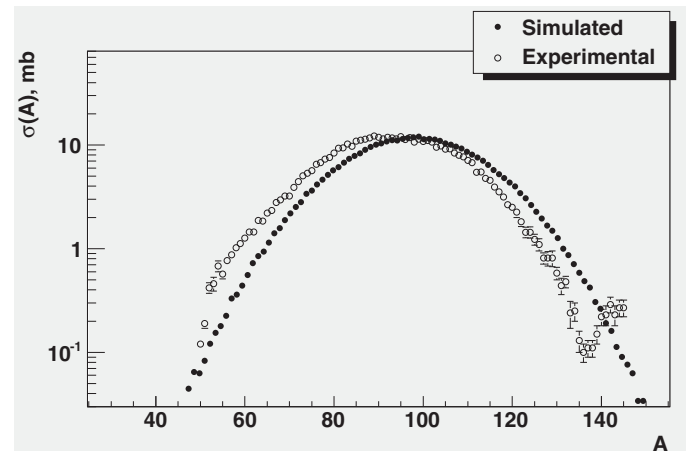

Fig. 7. Fragment-mass distribution for fission induced by $500 \mathrm{MeV}$ protons on ${ }^{208} \mathrm{~Pb}$ as simulated by CRISP (•). Experimental data are from GSI [36] (o).

The results presented in Figure 7 show a fair agreement between calculation and experiment. The calculated distribution reproduces the shape of the experimental distribution remarkably well, but there is a shift of $9.82 \pm 0.05$ mass units of the overall distribution toward the high mass region. This shift could be corrected by including the emission of prompt neutrons during the fission process and including the evaporation of nucleons and clusters from the fission fragments.

There is an apparent puzzle in the conclusions obtained so far, since using the symmetric fission dominance prescription we do not reproduce the results for actinide nuclei, but have much better 
EPJ Web of Conferences

Table 2. Values of the relevant parameters found for the best agreement between simulated and experimental data for the fission of ${ }^{208} \mathrm{~Pb}$ induced by $500 \mathrm{MeV}$ protons. Errors indicated represent superior limit for uncertainties.

\begin{tabular}{ll}
\hline parameter & value \\
\hline$a_{1}$ & $0.01 \pm 0.05$ \\
$a_{2}$ & $121.68 \pm 0.05$ \\
$a_{3}$ & $0.23 \pm 0.05$ \\
$a_{4}$ & $125.66 \pm 0.05$ \\
$a_{5}$ & $14.93 \pm 0.05$ \\
$a_{6}$ & $3.97 \pm 0.05$ \\
$a_{7}$ & $5.21 \pm 0.05$ \\
\hline
\end{tabular}

results for lead. However, the systematics at low energies [6] already shows that for nuclei with mass numbers around 220 the symmetric mode is dominante. Therefore one could suppose that fission at those excitation energies are still sensitive to the nuclear structure.

Of course we do not claim here that this is the case, since more precise results for actinide fission fragment mass distributions at high energies are necessary in order to extract informations about the fission dynamics.

\section{References}

1. Brosa U, Grossmann S and Muller A Phys. Rep. 197 (1990) 167-262.

2. Kudo H, Maruyama M, Tanikawa M, Shinozuka T and Fujioka M Phys. Rev. C 57 (1998) 178-188.

3. Wagemans C, Schillebeeckx P and Deruytter A Nucl. Phys. A 502 (1989) 287-296.

4. Weber Th, et al. Nucl. Phys. A 502 (1989) 279-286.

5. Sida J L, et al. Nucl. Phys. A 502 (1989) 233-242.

6. Bockstiegel C, Steinhauser S, Schimdt K -H, Clerc H -G, Grewe A, Heinz A, de Jong M, Junghans A R, Muller J and Voss B Nucl. Phys. A 802 (2008) 12-25.

7. Hambsch F J, Oberstedt S, Vladuca G and Tudora A Nucl. Phys. A 709 (2002) 85-102.

8. Hambsch F J, et al. Nucl. Phys. A 726 (2003) 248-264.

9. Weigmann H, Knitter H H and Hambsch F J Nucl. Phys. A 502 (1989) 177-194.

10. Ohtsuki T, et al. Phys. Rev. C 40 (1989) 2144-2153.

11. Duijvestijn M C, et. al. Phys. Rev C 59 (1999) 776-788.

12. Maslov V M Nucl. Phys. A 717 (2003) 3-20.

13. Itkis M G, Okolovich V N, Rusanov A Ya and Smirenkin G N Z. Phys. A 320 (1985) 433-441.

14. Pokrovsky I V, et al. Phys. Rev. C 62 (2000) 1-10.

15. Demekhina N A and Karapetyan G S Phys. At. Nucl. 71 (2008) 27-35.

16. Karapetyan G S, Balabekyan A R, Demekhina N A and Adam J Phys. At. Nucl. 72 (2009) 911-916.

17. Andrade- E. II; Menezes J. C. M.; Duarte S. B.; et al. Journal Phys. G: Nucl. and Part. 38 (2011) 085104.

18. E Andrade-Ii et al J. Phys.: Conf. Ser. 312 (2011) 082010.

19. Terranova M.L. and Tavares O.A.P. Phys. Scripta 49 (1994) 267-279.

20. Deppman A, Duarte S B, Silva G, Tavares O A P, Anefalos S, Arrudo-Neto J D T and Rodrigues T E J. Phys. G: Nucl. Part. Phys 30 (2004) 1991-2003.

21. Kodama T, Duarte S B, Chung K C, Nazareth R A M S Phys. Rev. Lett. 49 (1982) 536-539.

22. Goncalves M, dePina S, Lima D A, Milomen W, Medeiros E L and Duarte S B Phys. Lett. B 406 (1997) $1-6$.

23. Deppman A, Tavares O A P, Duarte S B, de Oliveira E C, Arruda-Neto J D T, de Pina S R, Likhachev V P, Rodriguez O, Mesa J and Goncalves M Phys. Rev. Lett. 87 (2001) 1-4

24. de Pina S et al. Phys. Lett. B 434 (1998) 1-6.

25. Deppman A, Tavares O A P, Duarte S B, de Oliveira E C, Arruda-Neto J D T, de Pina S R, Likhachev V P, Rodriguez O, Mesa J and Goncalves M Comp. Phys. Comm 145 (2002) 385-394. 


\section{$\mathrm{CNR} * 11$}

26. Deppman A, Tavares O A P, Duarte S B, Arruda-Neto J D T, Goncalves M, Likhachev V P and de Oliveira E C Phys. Rev. C 66 (2002) 067601.

27. Deppman A, Silva G, Anefalos S, Duarte S B, Garcia F, Hisamoto F H and Tavares O A P Phys. Rev. C 73 (2006) 1-5.

28. Andrade-II E, Freitas E, Tavares O A P, Duarte S B, Deppman A and Garcia F Proc. Conf. on XXXI Worshop on Nuclear Physics in Brazil vol 1139 (American Institute of Physics) (2009) p 64-69.

29. S.A. Pereira et al., Nuc. Sci. and Eng. 159 (2008) p.102-105.

30. Anefalos S, Deppman A, Silva G, Maiorino J R, dos Santos A, Duarte S B, Tavares O A P and Garcia F Braz. J. Phys. 35 (2005) 912-914.

31. Anefalos S, Deppman A, Arruda-Neto J D T, da Silva G, Maiorino J R, dos Santos A and Garcia F Int. Conf. on Nuclear Data for Science and Technology 769 (2004) 1299-1302.

32. Mongelli S T, Maiorino J R, Anefalos S, Deppman A and Carluccio T Braz. J. Phys. 35 (2005) 894-897.

33. Gonzales I, Barbero C, Deppman A, Duarte S B, Krmpotic F and Rodriguez O J. Phys. G: Nucl. Part. Phys. 38 (2011) 115105

34. Gonzalez I et al J. Phys.: Conf. Ser. 312 (2011) 022017.

35. Siegler P, Hambsch F -J, Oberstedt S and Theobald J P Nucl. Phys. A 594 (1995) 45-56.

36. Fernandez-Dominguez B et al. Nucl. Phys. A 747 (2005) 227-267. 